\title{
GaN nanowires with pentagon shape cross-section by ammonia- source molecular beam epitaxy
}

\author{
Yong Lin, Benjamin Leung, Qiming Li, Jeffrey. J. Figiel, and George T. Wang* \\ Sandia National Laboratories, Albuquerque, New Mexico 87185
}

\begin{abstract}
Ammonia-based molecular beam epitaxy $\left(\mathrm{NH}_{3}-\mathrm{MBE}\right)$ was used to grow catalyst-assisted $\mathrm{GaN}$ nanowires on (1102) r-plane sapphire substrates. Dislocation free [1120] oriented nanowires are formed with pentagon shape cross-section, instead of the usual triangular shape facet configuration. Specifically, the cross-section is the result of the additional two nonpolar $\{1010\}$ side facets, which appear due to a decrease in relative growth rate of the $\{1010\}$ facets to the $\{1011\}$ and $\{1011\}$ facets under the growth regime in $\mathrm{NH}_{3}-\mathrm{MBE}$. Compared to GaN nanowires grown by Ni-catalyzed metal-organic chemical vapor deposition, the $\mathrm{NH}_{3}-\mathrm{MBE}$ grown $\mathrm{GaN}$ nanowires show more than an order of magnitude increase in band-edge to yellow luminescence intensity ratio, as measured by cathodoluminescence, indicating improved microstructural and optical properties.
\end{abstract}

\footnotetext{
*Author to whom correspondence should be addressed: Electronic mail: gtwang@ sandia.gov.
} 


\section{Introduction}

The potential applications of III-nitride based nanowires in nanoscale electronic and optoelectronic devices have led to the development of an extensive range of synthesis methods. These growth techniques include metal-catalyzed metal-organic chemical vapor deposition (MOCVD) by the vapor-liquid-solid (VLS) mechanism, spontaneous formation in molecular beam epitaxy (MBE), and selective area growth by both MBE and MOCVD [1]-[11]. Even when using the same type of growth process, the resulting nanostructures can vary greatly in terms of geometry, morphology, crystallographic direction and defect content, leading to widely varying optical and electrical properties. These are determined by the specific details in the synthesis, such as the type, size and uniformity of the metal catalyst (if used), substrate type and orientation, precursor and carrier gases, and growth conditions (temperature, pressure, V/III ratio) [11]-[13]. Specifically, GaN nanowires have been reported by Ni catalyzed MOCVD to have a-axis or maxis orientation grown on r-plane sapphire substrate with triangular cross-sections [5], [8], [14][16]. There have also been several reports of trapezoidal or pentagonal non-polar axis nanowires [17], [18]. On the other hand, VLS and non-catalyzed CVD and MBE grown nanowires on cplane sapphire and Si (111) exhibit c-plane oriented nanowires with hexagonal cross-sections [1], [9], [10], [19]. The shape of the nanowire has been shown to be a crucial consideration in the fabrication of integrated single-nanowire devices. The different shapes imply unique facet combinations, especially important to the III-nitrides which possess strongly anisotropic polarization fields [15], [20]-[22]. These different orientations of (Al,Ga,In)N heterostructures possess strikingly different electrical and optical properties. In addition, the shape of nanowires has been a long standing consideration for device integration, especially for the metal contact formation to nanowires [23], [24].

One of the important advantages of the limited cross-sectional area of nanowires is that it enables dislocation free material to be observed, which gives an advantage especially for GaN 
material over its planar form. On the other hand, the impurity and point defect density can vary greatly in nanowires and may exceed that of standard thin films of GaN material. Luminescence studies on CVD nanowires show impurity incorporation and point defect formation likely caused by low VLS growth temperature and leading to the prevalence of the yellow luminescence (YL) band with a decreased band-edge luminescence (BEL) [8], [12], [25]. Improving the optical and electrical properties by reduction of these defects will enable the utilization of these nanoscale building blocks for high-performance optoelectronic devices.

Recently ammonia-based molecular beam epitaxy $\left(\mathrm{NH}_{3}-\mathrm{MBE}\right)$ has attracted attention for III-nitride growth owing to its advantages over currently widely used plasma-assisted MBE (PA$\mathrm{MBE}$ ), such as improved uniformity, larger growth window, reduced defect densities, and higher growth rates [26]. Specifically for III-nitride nanostructures, it has been reported for PA-MBE that high energy active nitrogen species supplied from the RF plasma source can damage the growing GaN nanostructures, resulting in microstructural defects associated with voids caused by sputter damage and dislocation generation [27]. With a gas source growth process supplying active nitrogen species by surface-catalyzed pyrolysis, such as $\mathrm{NH}_{3}-\mathrm{MBE}$, the high energy plasma source to activate nitrogen is not needed, and the luminescence properties are expected to improve. In addition, the growth windows that can be used in $\mathrm{NH}_{3}-\mathrm{MBE}$ have been shown to be much larger than for PA-MBE, so that a wider range growth regimes can be accessed [28], [29]. Particularly, this enables the use of higher temperatures than typical with MBE, with increased metal adatom surface mobility and reduced impurity incorporation. However, to date only a few reports in literature report synthesis of $\mathrm{GaN}$ nanowires using $\mathrm{NH}_{3}-\mathrm{MBE}$ [30]-[33]. In particular, the mapping of the growth window from planar to nanowire growth is of particular interest. As has been seen with PA-MBE, optimal nanowire growth conditions can differ significantly from planar growth conditions, such as the use of N-rich conditions for nanowires instead of near stoichiometric ones typically used for planar growths. In the growth window we find which 
results in Ni-catalyzed VLS growth of GaN nanowires with $\mathrm{NH}_{3}-\mathrm{MBE}$, nanowires are observed to have significant reduction of impurity incorporation and point defect formation as measured by a substantially improved BEL to YL ratio of a-axis oriented NWs. This growth method produces a pentagon-shaped cross-sectional nanowire morphology, enabling the use of a different facet combination and device geometry.

\section{Experimental Details}

All GaN nanowire samples were grown on 2-inch diameter (1102) r-plane sapphire substrates. Using electron-beam evaporation, a $3 \AA$ thick nickel (Ni) film was deposited onto sapphire substrates, to act as a metal catalyst, prior to insertion into the MBE chamber for GaN nanowire growth. Prior to the growth, Ni-coated sapphire substrates were annealed at $800^{\circ} \mathrm{C}$ in ultrahigh vacuum $\left(\leq 1 \times 10^{-9}\right.$ Torr) for 15 minutes. High-purity (99.999\%) ammonia was used as the nitrogen source and was introduced into the MBE chamber through a leak valve. No liquid nitrogen was circulating in the MBE cryopanel system during nanowire growths to shorten the post-growth MBE chamber pressure recovery time. During GaN nanowire growths, the $\mathrm{Ga}$ deposition rate used was $\sim 1 \AA / s$ and the ammonia over-pressure was maintained at $1 \times 10^{-4}$ Torr. Reduced ammonia over-pressure (to $5 \times 10^{-5}$ Torr) resulted in a decrease of vertical nanowire density. A substrate temperature of $900^{\circ} \mathrm{C}$ was selected for all $\mathrm{GaN}$ nanowire growths. We note that this temperature is considerably higher than those conventionally employed in $\mathrm{MBE}$ nanowire growth $\left(<825^{\circ} \mathrm{C}\right)$ [11], and could be reached without significant decomposition and desorption due to the growth window increase for $\mathrm{NH}_{3}$-MBE. All samples studied in this work have a growth time of 5 hours. Field emission scanning electron microscopy (SEM, FEI Quanta 200) was employed to study structural and morphological properties of GaN nanowires. In addition, crystalline and optical properties of individual nanowires were examined using transmission electron microscopy (TEM, JEOL 2010) and cathodoluminescence (CL, Gatan). The TEM and CL samples were prepared by scraping the surface of the as-grown GaN nanowire 
samples, to disperse the nanowires on copper grids (with carbon membranes) and silicon wafers, respectively.

\section{Results and Discussion}

The results of the Ni-catalyzed $\mathrm{NH}_{3}-\mathrm{MBE}$ grown nanowires are shown in Fig 1 . The GaN nanowires are uniformly distributed over the 2-inch sapphire substrates. Most of the nanowires are vertically aligned with respect to the substrate, while a fraction of the nanowires are tilted, as shown in Fig 1(a). The vertical nanowires have an average diameter of $640 \mathrm{~nm}$ (with a standard deviation of $80 \mathrm{~nm}$ ) at the base, and taper to a point along the nanowire length. The length of GaN nanowires is on average $\sim 5 \mu \mathrm{m}$ (with $1 \mu \mathrm{m}$ standard deviation), indicating a growth rate of $\sim 1 \mu \mathrm{m} / \mathrm{hr}$. and aspect ratios of $\sim 8$, extending the range of geometries achieved from the previously demonstrated nanowires by $\mathrm{NH}_{3}$-MBE.[11], [30]. The tapering mechanism of VLS grown nanowires has been studied extensively, and is determined by the migration of the metal catalyst. Under certain conditions, the metal catalyst has been observed to wet the nanowire sidewall, which eventually consumes the catalyst and terminates growth [34]-[36]. This effect has been shown to be reduced with decreased temperature [34], thus there is a tradeoff here between the amount of taper tolerable with the improved luminescence properties of the nanowires at higher temperatures.

The vertical nanowire density is estimated to be $\sim 7 \times 10^{7} / \mathrm{cm}^{2}$, and the percentage of tilted wires of all nanowires is approximately $15 \%$. The tilted nanowires are measured to have an inclination angle of $\sim 60^{\circ}$ from vertical. As is confirmed by TEM (Fig 2), the vertical nanowires are a-axis oriented. The tilted nanowires are tilted perpendicular to the GaN c-axis, which also give these wires an a-axis orientation from the equivalent $60^{\circ}$ inclined plane. The proportions of these tilted wires that persist after this growth length agree well with the previously performed Monte Carlo simulations, with the observed vertical nanowire density [14]. Thus the nucleation 
of the two orientations of nanowires begins with approximately equal probability, which rapidly favor the persistence of vertical nanowire growth through the collision mechanism. The remnants of the nucleated tilted wires are seen from the numerous GaN crystals at the base of the wires.

The cross-section of the nanowires grown here by $\mathrm{NH}_{3}-\mathrm{MBE}$ are neither the triangular or hexagonal shapes that are usually reported for GaN nanowires [1], [4]-[6], [8], [19], but a distinct pentagon shape, as shown in Fig 1(c). This observation strongly suggests that the nanowire crosssection is not only dependent on the crystallographic alignment with the substrate, but is also affected by growth conditions. In previous CVD experiments resulting in altered cross-sectional shape, the effects of carrier gases were attributed to the morphological change, in particular the facet dependent growth rate of $\mathrm{GaN}$ due to $\mathrm{H}_{2}$ gas [17]. Here, it may be possible that the decomposition of $\mathrm{NH}_{3}$ increases the active $\mathrm{H}$ species in the growth process, however previous MOCVD growths conducted under pure $\mathrm{H}_{2}$ gas have also resulted in triangular cross-sections [8], [12]. To gain a better understanding of the growth condition dependence on facet growth rates which we require to explain the formation of additional sidewall facets on these nanowires, we draw on experiments performed with MOCVD and HVPE with selective area growth [21], [37]. We note that these studies, drawing on the crystal growth principles that govern the facet evolution during deposition, can describe the appearance of facets in non-equilibrium $\mathrm{GaN}$ growth processes by the kinetic Wulff plot, or v-plot. These principles have been used previously in nanowire growths [38] to explain and predict differing facet structures. The facets that appear on these nanowires are determined to be the (0001) Ga-polar or (0001) N-polar facet, two perpendicular $\{1010\}$ side facets, and two bounding $\{1011\}$ or $\{1011\}$ facets, as measured from the facet angles and orientation of the nanowire by top view and side view SEM images, Fig 1 (b) and (c), and TEM, Fig 2. Here, the exact polarity of each nanowire is not determined, i.e. differentiation between (0001) and (0001). This is because orientations of the pentagonal shape are observed in both directions with respect to the substrate. A change in epitaxial relation has 
previously shown to not occur for non-polar GaN on r-sapphire [39], thus it is most likely that both sets of facets exist. As the nanowire is tapered, the (0001) and (0001) side of the pentagon consists of terraced facets along the length of the nanowire. The difference giving the pentagon shape shown here and the typically observed triangular shape for a-axis oriented GaN nanowires is the additional appearance of the $\{1010\}$ side facets. In the cases where the triangular shape is obtained (MOCVD, HVPE), the $\{1010\}$ growth rate exceeds that of the projected growth of the bounding $\{1011\},\{1011\}$ and (0001) facets, and thus extends to extinction. This is consistent with what is commonly observed in these growth methods, that the (0001) and $\{1011\}$ facets are always the slowest growth rate facets in selective area growth experiments [21], [40], [41]. Here using $\mathrm{NH}_{3}-\mathrm{MBE}$, the growth conditions differ significantly with respect to these growth methods. As observed, this results in kinetically limiting the growth rate of the $\{1010\}$ facets, leading to the appearance of these facets in these nanowires. The decrease in relative growth rate of the $\{101$ $0\}$ facet to the $\{1011\}$ facet tends to occur at higher temperatures with lower V/III ratio growth conditions, as has been reported [21], [41]. This is consistent with the growth regime we are operating at with the $\mathrm{NH}_{3}-\mathrm{MBE}$ growth process, as compared to MOCVD and PA-MBE.

Figure 2 shows a bright field TEM image of a nanowire, with a zone axis of [0001]. The corresponding selected area diffraction pattern of the single GaN nanowire is shown in the inset. The growth direction of the GaN nanowires is determined to be [1120] by the means of aligning the TEM image and the diffraction pattern. It is noted that this growth direction is the same as that grown on r-plane sapphire by Ni-catalyzed MOCVD [8], despite the differing nanowire geometry. No dislocation contrast can be seen, consistent with the typical result of dislocationfree VLS GaN nanowire growth.

To probe the point defect content and evaluate the optical properties of these nanowires, room temperature CL measurements were carried out. Fig 3(a) shows the measured CL spectrum integrated over the nanowire volume, in which a GaN band-edge recombination peak at $369 \mathrm{~nm}$ 
dominates and nearly no YL can be detected. As a comparison, a typical CL spectrum of MOCVD grown Ni-catalyzed GaN nanowires on r-plane sapphire substrates is shown in Fig 3(b). The detailed growth process for these nanowires can be found in previous reports [8], [12]. In contrast, these nanowires display a distinct YL band at $\sim 560 \mathrm{~nm}$. The BEL/YL ratio, which indicates the optical quality of the material, is approximately 2. For the $\mathrm{NH}_{3}-\mathrm{MBE}$ grown nanowires demonstrated here, the BEL/YL ratio increases by almost two orders of magnitude, to 150. We note that further investigation is needed to determine the cause of the larger width of the BEL peak of the $\mathrm{NH}_{3}$-MBE grown wires than that of the MOCVD grown wires.

YL in GaN-related materials are attributed to electronic transitions from the conduction or shallow donor states to a deep acceptor [42]-[46]. The candidates for the deep acceptor have been investigated extensively, and are associated with either carbon impurities, or Ga-vacancies $\mathrm{V}_{\mathrm{Ga}}$ and its oxygen complex $\mathrm{V}_{\mathrm{Ga}} \mathrm{O}_{\mathrm{N}}$. Particularly, for $\mathrm{GaN}$ nanowires, spatially resolved measurements of the YL as a function of nanowire diameter have suggested that the YL-related defects initially forms through a combination of VLS and sidewall growth, and subsequently redistributes towards the surface by diffusion [12]. The mobility of these point defects indicates the role of isolated $\mathrm{V}_{\mathrm{Ga}}$ that diffuse and pile up at the surface. A background concentration of $\mathrm{V}_{\mathrm{Ga}} \mathrm{O}_{\mathrm{N}}$ complexes has also been suggested in MBE grown nanowires [47]. The reduction of these defects in $\mathrm{NH}_{3}$-MBE may be attributed to the increase metal adatom surface mobility due to high growth temperatures and elimination of high energy N species. Since no intentional C sources are introduced in these $\mathrm{NH}_{3}-\mathrm{MBE}$ growths, a decrease in carbon impurities and $\mathrm{V}_{\mathrm{Ga}} \mathrm{C}_{\mathrm{N}}$ point defects from MOCVD grown wires is also a likely possibility. Through these measurements, it is demonstrated that $\mathrm{NH}_{3}-\mathrm{MBE}$ is a useful method in producing high crystalline quality $\mathrm{GaN}$ nanostructures with excellent optical properties.

\section{Conclusion}


In summary, $\mathrm{GaN}$ nanowires have been grown on r-plane sapphire substrates using $\mathrm{NH}_{3}-$ MBE. It was observed for the first time that GaN nanowires grown by this approach have a distinct pentagon cross-section, which is believed to closely relate to both the substrate and growth conditions. Using TEM, the growth direction of GaN nanowires was determined to be [1120], identical to that of GaN nanowires grown on r-plane sapphire substrates using MOCVD. $\mathrm{CL}$ results demonstrate that $\mathrm{GaN}$ nanowires by ammonia-based MBE have superior optical properties as evidenced by a substantially increased BEL to YL ratio than that of MOCVD-grown samples.

\section{Acknowledgements}

This work is supported by DOE Basic Energy Sciences and Sandia's Laboratory Directed Research and Development program. Sandia National Laboratories is a multi-program laboratory managed and operated by Sandia Corporation, a wholly owned subsidiary of Lockheed Martin Corporation, for the U.S. Department of Energy's National Nuclear Security Administration under contract DE-AC04-94AL85000. 


\section{References}

[1] K. A. Bertness, A. Roshko, N. A. Sanford, J. M. Barker, and A. V. Davydov, "Spontaneously grown GaN and AlGaN nanowires," J. Cryst. Growth, vol. 287, no. 2, pp. 522-527, Jan. 2006.

[2] R. Calarco, R. J. Meijers, R. K. Debnath, T. Stoica, E. Sutter, and H. Lüth, "Nucleation and Growth of GaN Nanowires on Si(111) Performed by Molecular Beam Epitaxy," Nano Lett., vol. 7, no. 8, pp. 2248-2251, Aug. 2007.

[3] X. Duan and C. M. Lieber, "Laser-Assisted Catalytic Growth of Single Crystal GaN Nanowires," J. Am. Chem. Soc., vol. 122, no. 1, pp. 188-189, Jan. 2000.

[4] M. He, I. Minus, P. Zhou, S. N. Mohammed, J. B. Halpern, R. Jacobs, W. L. Sarney, L. Salamanca-Riba, and R. D. Vispute, "Growth of large-scale GaN nanowires and tubes by direct reaction of Ga with NH[sub 3]," Appl. Phys. Lett., vol. 77, no. 23, p. 3731, 2000.

[5] T. Kuykendall, P. Pauzauskie, S. Lee, Y. Zhang, J. Goldberger, and P. Yang, "Metalorganic Chemical Vapor Deposition Route to GaN Nanowires with Triangular Cross Sections," Nano Lett., vol. 3, no. 8, pp. 1063-1066, Aug. 2003.

[6] H. . Peng, X. . Zhou, N. Wang, Y. . Zheng, L. . Liao, W. . Shi, C. . Lee, and S. . Lee, "Bulk-quantity GaN nanowires synthesized from hot filament chemical vapor deposition," Chem. Phys. Lett., vol. 327, no. 5-6, pp. 263-270, Sep. 2000.

[7] G. Suo, S. Jiang, J. Zhang, J. Li, and M. He, "Synthetic Strategies and Applications of GaN Nanowires," Adv. Condens. Matter Phys., vol. 2014, pp. 1-11, 2014.

[8] G. T. Wang, A. A. Talin, D. J. Werder, J. R. Creighton, E. Lai, R. J. Anderson, and I. Arslan, "Highly aligned, template-free growth and characterization of vertical GaN nanowires on sapphire by metal-organic chemical vapour deposition," Nanotechnology, vol. 17, no. 23, pp. 5773-5780, Dec. 2006.

[9] S. D. Hersee, X. Sun, and X. Wang, "The Controlled Growth of GaN Nanowires," Nano Lett., vol. 6, no. 8, pp. 1808-1811, Aug. 2006.

[10] H. Sekiguchi, T. Nakazato, A. Kikuchi, and K. Kishino, "Structural and optical properties of GaN nanocolumns grown on (0001) sapphire substrates by rf-plasmaassisted molecular-beam epitaxy," J. Cryst. Growth, vol. 300, no. 1, pp. 259-262, Mar. 2007.

[11] K. A. Bertness, N. A. Sanford, and A. V. Davydov, "GaN Nanowires Grown by Molecular Beam Epitaxy,” IEEE J. Sel. Top. Quantum Electron., vol. 17, no. 4, pp. 847-858, Jul. 2011.

[12] Q. Li and G. T. Wang, "Spatial Distribution of Defect Luminescence in GaN Nanowires," Nano Lett., vol. 10, no. 5, pp. 1554-1558, May 2010.

[13] T. R. Kuykendall, V. Altoe, D. F. Ogletree, and S. Aloni, "Catalyst-Directed Crystallographic Orientation Control of GaN Nanowire Growth,” Nano Lett., p. 141112122104007 , Nov. 2014.

[14] Q. Li, J. R. Creighton, and G. T. Wang, "The role of collisions in the aligned growth of vertical nanowires," J. Cryst. Growth, vol. 310, no. 16, pp. 3706-3709, Aug. 2008.

[15] F. Qian, S. Gradec $\square$ ak, Y. Li, C.-Y. Wen, and C. M. Lieber, "Core/Multishell Nanowire Heterostructures as Multicolor, High-Efficiency Light-Emitting Diodes," Nano Lett., vol. 5, no. 11, pp. 2287-2291, Nov. 2005. 
[16] T. Aschenbrenner, C. Kruse, G. Kunert, S. Figge, K. Sebald, J. Kalden, T. Voss, J. Gutowski, and D. Hommel, "Highly ordered catalyst-free and mask-free GaN nanorods on $r$-plane sapphire," Nanotechnology, vol. 20, no. 7, p. 075604, Feb. 2009.

[17] C. Chen, T. Yan, W. Hsiang, and M. M. C. Chou, "Morphological control over nonpolar GaN nanostructures on (100) $\gamma$-LiAlO2 substrate," Mater. Lett., vol. 130, pp. 271-273, Sep. 2014.

[18] B. Zhang, H. Song, X. Xu, J. Liu, J. Wang, X. Liu, S. Yang, Q. Zhu, and Z. Wang, "Well-aligned Zn-doped tilted InN nanorods grown on $r$-plane sapphire by MOCVD," Nanotechnology, vol. 22, no. 23, p. 235603, Jun. 2011.

[19] L. W. Tu, C. L. Hsiao, T. W. Chi, I. Lo, and K. Y. Hsieh, "Self-assembled vertical GaN nanorods grown by molecular-beam epitaxy," Appl. Phys. Lett., vol. 82, no. 10, p. 1601, 2003.

[20] T. Wernicke, L. Schade, C. Netzel, J. Rass, V. Hoffmann, S. Ploch, A. Knauer, M. Weyers, U. Schwarz, and M. Kneissl, "Indium incorporation and emission wavelength of polar, nonpolar and semipolar InGaN quantum wells," Semicond. Sci. Technol., vol. 27, no. 2, p. 024014, Feb. 2012.

[21] B. Leung, Q. Sun, C. D. Yerino, J. Han, and M. E. Coltrin, "Using the kinetic Wulff plot to design and control nonpolar and semipolar GaN heteroepitaxy," Semicond. Sci. Technol., vol. 27, no. 2, p. 024005, Feb. 2012.

[22] B. M. Wong, F. Léonard, Q. Li, and G. T. Wang, "Nanoscale Effects on Heterojunction Electron Gases in GaN/AlGaN Core/Shell Nanowires," Nano Lett., vol. 11, no. 8, pp. 3074-3079, Aug. 2011.

[23] F. Léonard and A. A. Talin, "Electrical contacts to one- and two-dimensional nanomaterials," Nat. Nanotechnol., vol. 6, no. 12, pp. 773-783, Nov. 2011.

[24] F. Qian, Y. Li, S. Gradečak, H.-G. Park, Y. Dong, Y. Ding, Z. L. Wang, and C. M. Lieber, "Multi-quantum-well nanowire heterostructures for wavelength-controlled lasers," Nat. Mater., vol. 7, no. 9, pp. 701-706, Sep. 2008.

[25] A. A. Talin, G. T. Wang, E. Lai, and R. J. Anderson, "Correlation of growth temperature, photoluminescence, and resistivity in GaN nanowires," Appl. Phys. Lett., vol. 92, no. 9, p. 093105, 2008.

[26] A. L. Corrion, C. Poblenz, F. Wu, and J. S. Speck, "Structural and morphological properties of $\mathrm{GaN}$ buffer layers grown by ammonia molecular beam epitaxy on $\mathrm{SiC}$ substrates for AlGaN/GaN high electron mobility transistors," J. Appl. Phys., vol. 103, no. 9, p. 093529, 2008.

[27] D. P. Norman, S. M. Hamad, L.-W. Tu, Y.-T. Lin, C.-Y. Lin, and H.-W. Seo, "Effects of plasma power on material and optical quality of GaN nanorods grown by plasma-assisted molecular beam epitaxy," Appl. Phys. Express, vol. 7, no. 11, p. 115502, Nov. 2014.

[28] A. L. Corrion, F. Wu, and J. S. Speck, "Growth regimes during homoepitaxial growth of GaN by ammonia molecular beam epitaxy," J. Appl. Phys., vol. 112, no. 5, p. 054903, 2012.

[29] A. N. Alexeev, V. P. Chaly, D. M. Krasovitsky, V. V. Mamaev, S. I. Petrov, and V. G. Sidorov, "Features and benefits of III-N growth by ammonia-MBE and plasma assisted MBE," J. Phys. Conf. Ser., vol. 541, p. 012030, Oct. 2014. 
[30] S. Vézian, B. Alloing, and J. Zúñiga-Pérez, "GaN nanocolumns on sapphire by ammonia-MBE: From self-organized to site-controlled growth," J. Cryst. Growth, vol. 323, no. 1, pp. 326-329, May 2011.

[31] B. Alloing, S. Vézian, O. Tottereau, P. Vennéguès, E. Beraudo, and J. ZunigaPérez, "On the polarity of GaN micro- and nanowires epitaxially grown on sapphire (0001) and $\mathrm{Si}(111)$ substrates by metal organic vapor phase epitaxy and ammoniamolecular beam epitaxy," Appl. Phys. Lett., vol. 98, no. 1, p. 011914, 2011.

[32] K. A. Bertness, A. Roshko, N. A. Sanford, J. B. Schlager, and M. H. Gray, "Formation of AlN and GaN nanocolumns on $\mathrm{Si}(111)$ using molecular beam epitaxy with ammonia as a nitrogen source," Phys. Status Solidi C, vol. 2, no. 7, pp. 23692372, May 2005.

[33] K. Kawasaki, I. Nakamatsu, H. Hirayama, K. Tsutsui, and Y. Aoyagi, "Formation of GaN nanopillars by selective area growth using ammonia gas source molecular beam epitaxy," J. Cryst. Growth, vol. 243, no. 1, pp. 129-133, Aug. 2002.

[34] Y. Wang, V. Schmidt, S. Senz, and U. Gösele, "Epitaxial growth of silicon nanowires using an aluminium catalyst," Nat. Nanotechnol., vol. 1, no. 3, pp. 186189, Dec. 2006.

[35] L. Cao, B. Garipcan, J. S. Atchison, C. Ni, B. Nabet, and J. E. Spanier, "Instability and Transport of Metal Catalyst in the Growth of Tapered Silicon Nanowires," Nano Lett., vol. 6, no. 9, pp. 1852-1857, Sep. 2006.

[36] J. B. Hannon, S. Kodambaka, F. M. Ross, and R. M. Tromp, "The influence of the surface migration of gold on the growth of silicon nanowires," Nature, vol. 440, no. 7080, pp. 69-71, Mar. 2006.

[37] D. Du, D. J. Srolovitz, M. E. Coltrin, and C. C. Mitchell, "Systematic Prediction of Kinetically Limited Crystal Growth Morphologies," Phys. Rev. Lett., vol. 95, no. 15, Oct. 2005.

[38] X. J. Chen, G. Perillat-Merceroz, D. Sam-Giao, C. Durand, and J. Eymery, "Homoepitaxial growth of catalyst-free GaN wires on N-polar substrates," Appl. Phys. Lett., vol. 97, no. 15, p. 151909, 2010.

[39] P. Vennegues, T. Zhu, Z. Bougrioua, D. Martin, J. Zuniga-Perez, and N. Grandjean, "In-Plane Polarities of Nonpolar Wurtzite Epitaxial Films Deposited on $m$ - and $r$-plane Sapphire Substrates," Jpn. J. Appl. Phys., vol. 48, no. 9, p. 090211, Sep. 2009.

[40] K. Hiramatsu, K. Nishiyama, M. Onishi, H. Mizutani, M. Narukawa, A. Motogaito, H. Miyake, Y. Iyechika, and T. Maeda, "Fabrication and characterization of low defect density GaN using facet-controlled epitaxial lateral overgrowth (FACELO)," J. Cryst. Growth, vol. 221, no. 1-4, pp. 316-326, Dec. 2000.

[41] K. Hiramatsu and H. Miyake, "Review of Facet Controlled Epitaxial Lateral Overgrowth (FACELO) of GaN via Low Pressure Vapor Phase Epitaxy," MRS Proc., vol. 639, Jan. 2000.

[42] T. Ogino and M. Aoki, "Mechanism of Yellow Luminescence in GaN," Jpn. J. Appl. Phys., vol. 19, no. 12, pp. 2395-2405, Dec. 1980.

[43] T. Suski, P. Perlin, H. Teisseyre, M. Leszczyński, I. Grzegory, J. Jun, M. Boćkowski, S. Porowski, and T. D. Moustakas, "Mechanism of yellow luminescence in GaN," Appl. Phys. Lett., vol. 67, no. 15, p. 2188, 1995. 
[44] E. Calleja, F. J. Sánchez, D. Basak, M. A. Sánchez-García, E. Muñoz, I. Izpura, F. Calle, J. M. G. Tijero, J. L. Sánchez-Rojas, B. Beaumont, P. Lorenzini, and P. Gibart, "Yellow luminescence and related deep states in undoped GaN," Phys. Rev. B, vol. 55, no. 7, pp. 4689-4694, Feb. 1997.

[45] H. Tang, J. B. Webb, J. A. Bardwell, S. Raymond, J. Salzman, and C. UzanSaguy, "Properties of carbon-doped GaN," Appl. Phys. Lett., vol. 78, no. 6, p. 757, 2001.

[46] S. O. Kucheyev, M. Toth, M. R. Phillips, J. S. Williams, C. Jagadish, and G. Li, "Chemical origin of the yellow luminescence in GaN," J. Appl. Phys., vol. 91, no. 9, p. 5867, 2002.

[47] Y. Kuo, "Reactive ion etching of plasma enhanced chemical vapor deposition amorphous silicon and silicon nitride: Feeding gas effects," J. Vac. Sci. Technol. Vac. Surf. Films, vol. 8, no. 3, p. 1702, May 1990. 

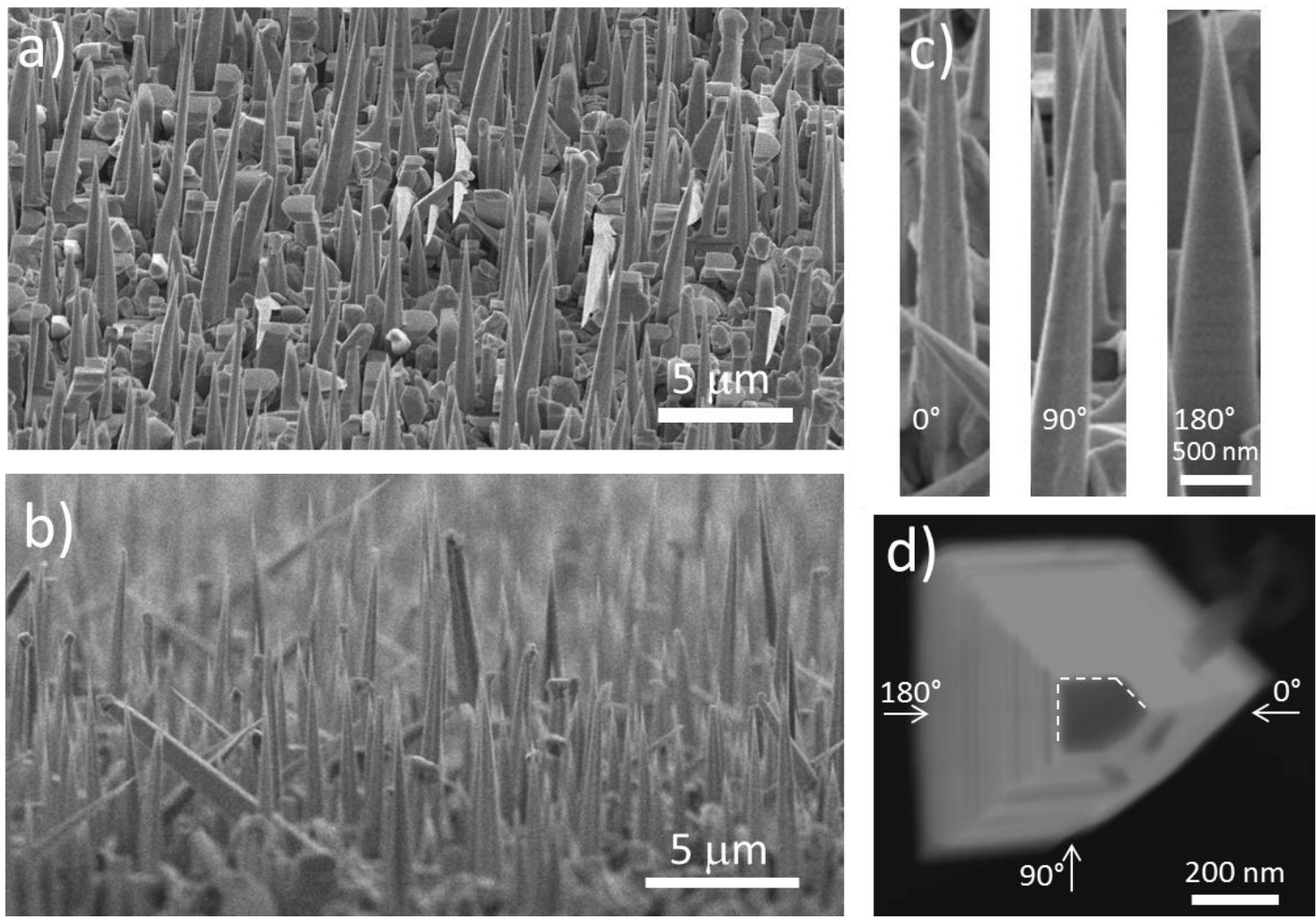

Figure 1. SEM images of $\mathrm{GaN}$ nanowires grown by $\mathrm{NH}_{3}-\mathrm{MBE}$ (a) Low magnification inclined view and (b)side view and (c) higher magnification side views of a single nanowire rotated from $0^{\circ}$ (in the [0001] direction), $90^{\circ}$ ([1010]) and $180^{\circ}$ ([0001]). (d) Top view, with the crystallographic facets marked (with the other facets identical by symmetry). The viewing angles of Fig 1(c) are marked. 


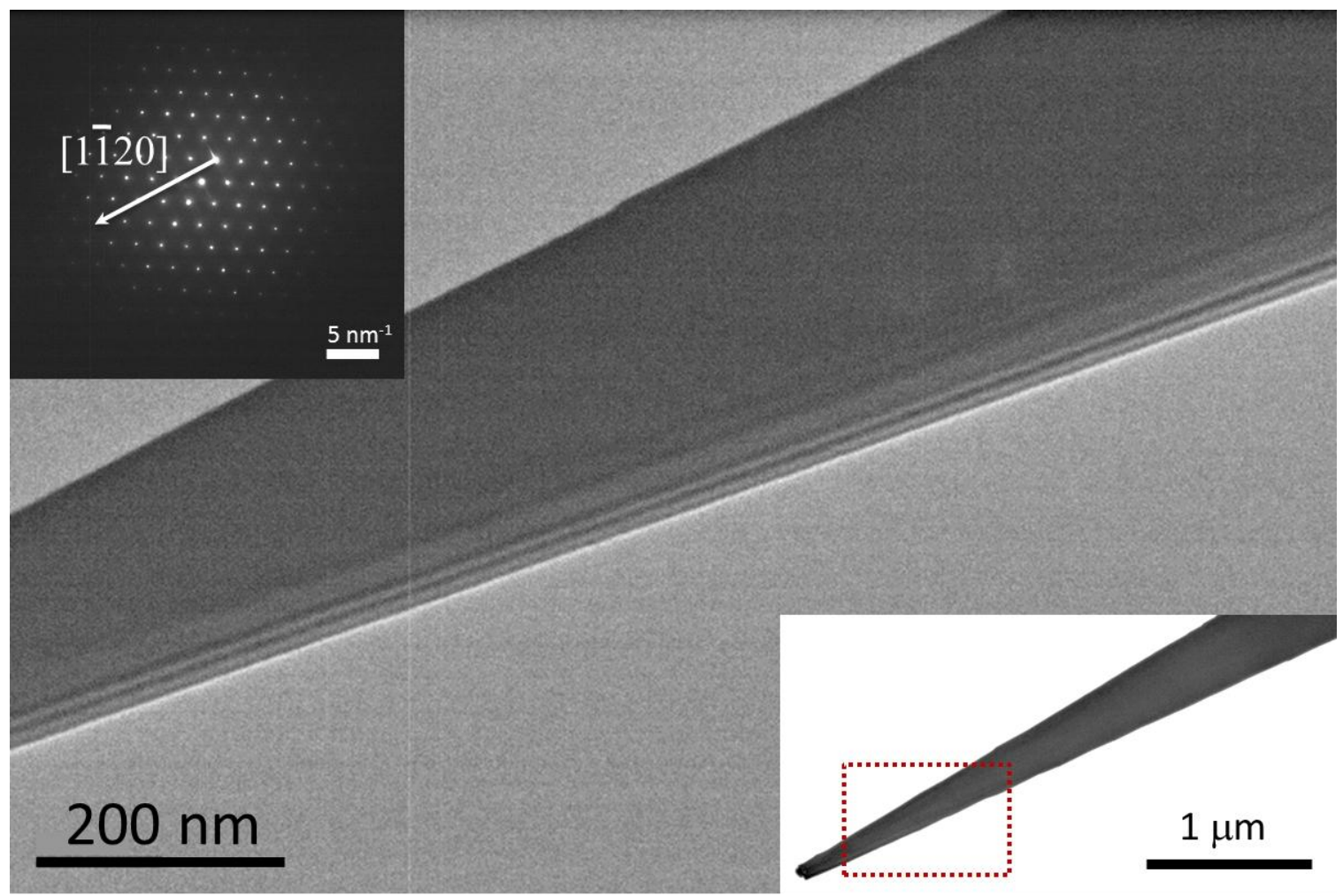

Figure 2. TEM image (zone axis of [0001]) of a single GaN nanowire and (inset) selected area diffraction pattern. 


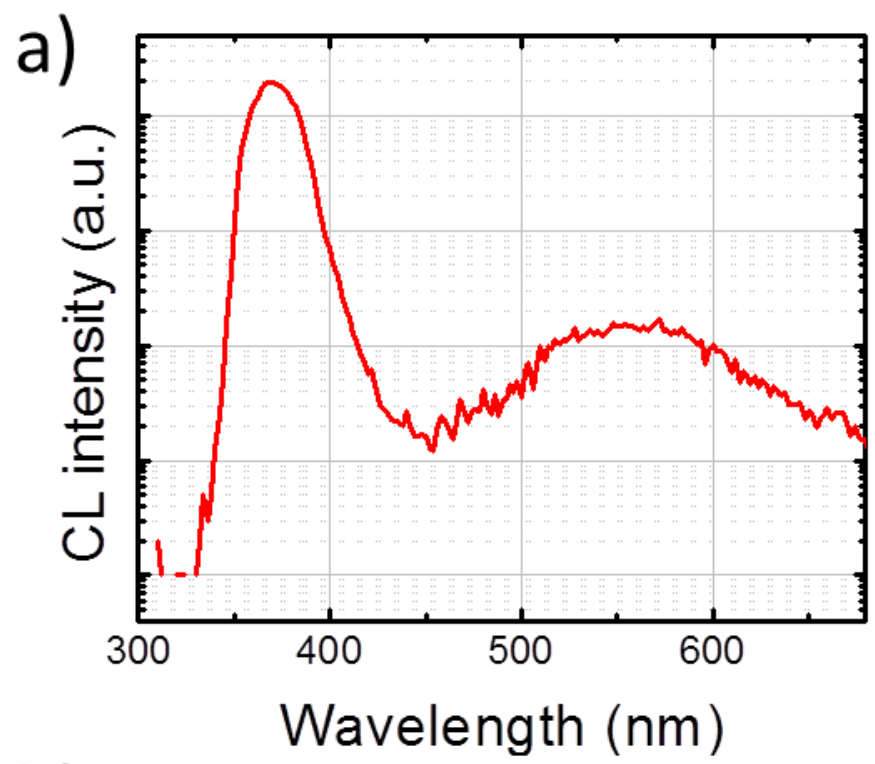

b)

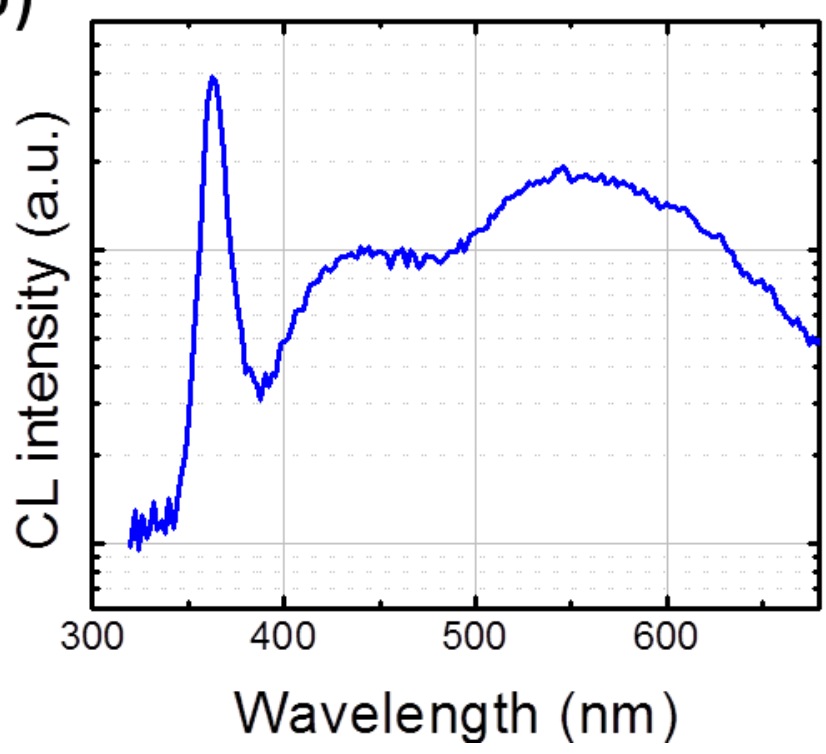

Figure 3. CL spectra of GaN nanowires grown on r-plane sapphire substrates by (a) $\mathrm{NH}_{3}-\mathrm{MBE}$ and (b) MOCVD. 